\title{
The role of indocyanine green fluorescence for intersegmental plane identification during video-assisted thoracoscopic surgery segmentectomies
}

\author{
Vadim G. Pischik ${ }^{1,2}$, Aleksandr Kovalenko ${ }^{1,2}$ \\ ${ }^{1}$ Department of Thoracic Surgery, Federal Hospital \#122, Saint Petersburg, Russia; ${ }^{2}$ Faculty of Medicine, Saint Petersburg State University, Saint \\ Petersburg, Russia \\ Contributions: (I) Conception and design: All authors; (II) Administrative support: None; (III) Provision of study materials or patients: All authors; \\ (IV) Collection and assembly of data: All authors; (V) Data analysis and interpretation: All authors; (VI) Manuscript writing: All authors; (VII) Final \\ approval of manuscript: All authors. \\ Correspondence to: Prof. Vadim G. Pischik, MD, PhD. 4 Prospect Kultury, Saint-Petersburg 194291, Russian. Email: vadim.pischik@mail.ru.
}

Background: According to recent studies, thoracoscopic segmentectomy is an acceptable alternative to lobectomy for treating different lung diseases. This approach appears to have equivalent rates of postoperative morbidity with potentially similar long-term results even in selected lung cancer patients. At the same time, surgical success is highly dependent on intraoperative interpretation of segmental anatomy. We aimed to analyze our lung segmentectomy experience and identify the role of indocyanine green (ICG)fluorescence for intersegmental plane detection.

Methods: A total of 86 consecutive patients who underwent 90 thoracoscopic segmentectomies with near-infrared-indocyanine green (NIR-ICG) method between September 2015 and December 2017 were investigated. According to the preoperative 3D lung hilar model, vascular and bronchial branches of the target segment were divided. ICG was thereafter injected into the central or peripheral vein. The boundary lines between the areas with and without fluorescence was marked on the visceral pleura by electrocautery.

Results: The fluorescence was detected immediately after bolus ICG injection in the central vein in all patients but had a time delay of 10-25 seconds in cases of peripheral vein administration. The median duration of intensive ICG staining was 90 seconds, regardless of the injection method. Well-defined fluorescence borders were observed in 86 of 90 (95.6\%) segmentectomies due to technical reasons in three cases and severe emphysema in one. Chronic obstructive pulmonary disease (COPD) in other patients did not impair the boundary line identification, but reduced the duration of intensive ICG staining. No ICGrelated complications were observed.

Conclusions: The ICG-fluorescence technology is safe and effective for verification of anatomic segment borders for video-assisted thoracoscopic surgery (VATS). The perfusion-based ICG fluorescence technique has advantages for thoracoscopic surgeries compared to other methods. Doubling the dose of ICG allows clear detection of the intersegmental plane, even in certain suboptimal conditions.

Keywords: Indocyanine green (ICG); near-infrared (NIR); thoracoscopic segmentectomy; fluorescence; intersegmental plane.

Submitted Feb 16, 2018. Accepted for publication Apr 09, 2018.

doi: $10.21037 /$ jtd.2018.04.84

View this article at: http://dx.doi.org/10.21037/jtd.2018.04.84 


\section{Introduction}

The growing interest in reducing surgical trauma and preserving lung function has resulted in an increasing need for thoracoscopic segmentectomy in recent years (1). According to recent studies, thoracoscopic segmentectomy is an acceptable alternative to lobectomy for treating different lung diseases. This approach appears to have equivalent rates of postoperative morbidity with potentially similar long-term results (2) even in selected cases of lung cancer (3). At the same time, surgical success is highly dependent on an intraoperative interpretation of segmental anatomy, especially for thoracoscopic procedures. Furthermore, precise identification of the intersegmental plane is one of the essential steps in segmentectomy. The commonly used air inflation techniques (4) may shift the border and also obstruct the view during video-assisted thoracoscopic surgery (VATS). Minimally-invasive surgery needed a novel method of augmented reality, which became possible by using near-infrared (NIR) fluorescence imaging with indocyanine green (ICG). This method is currently used in thoracic surgery for conduit vascular evaluation during esophagectomy (5), sentinel lymph nodes (LNs) mapping $(6,7)$, pulmonary nodule detection (8) and bullous lesion detection (9). However, publications describing the technique of identifying the lung intersegmental planes by NIR-ICG are still infrequent (10-14). We aimed to analyse our lung segmentectomy experience and identify the role of ICG-fluorescence for intersegmental plane detection.

\section{Methods}

The database from the Thoracic Surgery Department of Clinical Hospital \#122 in Saint Petersburg was used for the retrospective cohort study. The study was approved by ethics committee of Clinical Hospital №122 (No. 201527) and written informed consent was obtained from all patients. A total of 86 consecutive patients who underwent 90 thoracoscopic segmentectomies with NIR-ICG method between September 2015 and December 2017 were investigated. The mean age of patients was $55.2 \pm 18.1$ years (range, $21-78$ years), and $60 \%(\mathrm{n}=52)$ were female. None of the patients had allergic reactions to iodine-containing drugs. Written informed consent was obtained from all patients.

The preoperative workup included chest and abdominal computed tomography (CT) and/or integrated positron emission tomographic (PET) CT scanning, pulmonary function tests and cardiovascular examination. Forced expiratory volume in 1 second $\left(\mathrm{FEV}_{1}\right)$ used as a universal chronic obstructive pulmonary disease (COPD) marker, ranged from $32 \%$ to $95 \%$ of predicted value. The prevalence of severe COPD was higher in the heavy smoker group that included 16 patients (18\%). CT scans were also reconstructed in all patients as $3 \mathrm{D}$ images in order to define the location of the nodule and its relation to adjacent structures, pulmonary vessels, and bronchi. For this purpose, OsiriX MD software for Mac operating system, as well as Radiant DICOM Viewer for Windows were used.

Inclusion criteria for treating lung cancer were based on recent research data (1); patients with semisolid groundglass opacity (GGO) nodules less than $2 \mathrm{~cm}$ in diameter without mediastinal lymphadenopathy were included. Five patients who had tumors going beyond the criteria were scheduled to have a segmentectomy due to compromised pulmonary function. Candidates for metastasectomies were selected if the primary site of the disease was controlled and the lesion was located deep in lung parenchyma. Other indications for segmentectomies were small unspecified tumors and infectious lung diseases. One patient underwent two consecutive segmentectomies for bilateral arteriovenous malformations. Surgical treatment of three other patients required re-operation from the opposite side because of a new solitary lung metastasis. Four patients underwent segmentectomy after the previous lobectomy, in one case ipsilaterally.

A VATS approach was used to perform all segmentectomies, according to a previously reported technique (15), under general anaesthesia with single-lung ventilation and using double-ports access. The utility incision up to $3 \mathrm{~cm}$ was placed in the fourth or fifth intercostal space in the anterior axillary line and the trocar site was located in the seventh intercostal space in the mid-axillary line for $30^{\circ}$ thoracoscope (NIR ICGsystem, Karl Storz, Tuttlingen, Germany).

According to the preoperative 3D lung hilar model (Figure 1), vascular and bronchial branches of the target segment were isolated separately. The station 11 and station 12 LNs were dissected precisely with ultrasound scalpel (Figure 2). If the intraoperative examination of the LN by frozen section showed metastasis, the procedure was converted to lobectomy. Those patients were not included in the study.

After the division of segmental blood vessels and bronchi by endostapler or clips, ICG was injected into the central or peripheral vein. The initial dose of bolus administration was $0.15 \mathrm{mg}$ per $\mathrm{kg}$ body weight. Fluorescence immediately 


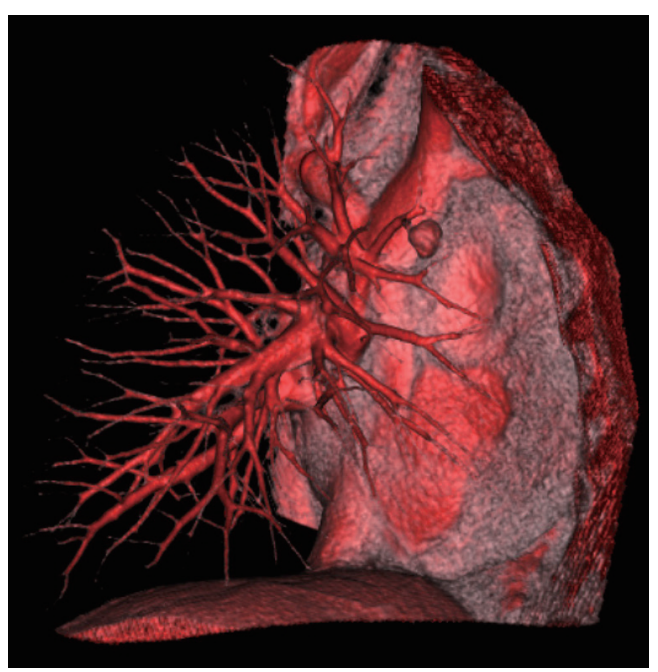

Figure 1 Preoperative 3D right lung hilar model (planned S3 segmentectomy). S, segment.

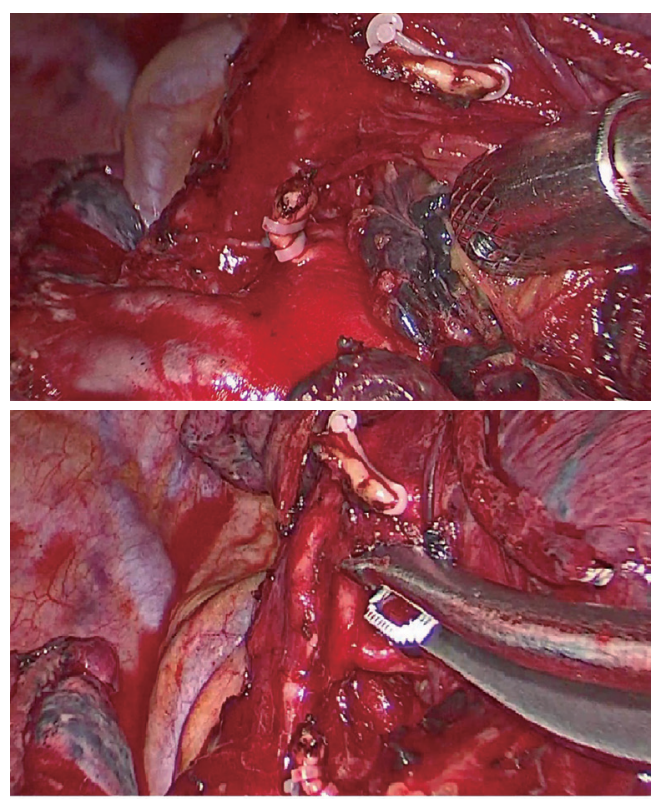

Figure 2 Intraoperative view before (top picture) and after (bottom picture) interlobar LN dissection (planned right S2 segmentectomy). S, segment; LN, lymph node.

covered all structures except the isolated target segment, which appeared dark (Figure 3). If the segmental anatomy or revealed fluorescence seemed doubtful, an additional ICG dose was administered.

The boundary line between the areas with and without fluorescence was marked on the visceral pleura by electrocautery. The intersegmental plane was then divided by endostaplers placed along the electrocautery line with the tips oriented toward the intersegmental veins. As a result, the stapling lines surrounded the peripheral stumps of pulmonary arteries, veins, and bronchi (Figure 4). In the surgical treatment of malignant diseases, segmentectomies were followed by lobe-specific lymphadenectomy of at least three LN stations, obligatorily including station 7 (Figure 5).

\section{Results}

Between September 2015 and December 2017, 86 patients (52 female) underwent planned thoracoscopic segmentectomy using ICG fluorescence with the following indications: 34 patients (40\%) for primary lung cancer, 30 patients (35\%) for single pulmonary metastasis (21 patients with colon carcinoma, 4 patients with papillary thyroid carcinoma, 3 patients with breast cancer, 1 patient with uterine leiomyoma and 1 patient with follicular lymphoma. Sixteen patients (19\%) received surgical treatment of infectious lesions (7 cases of tuberculoma, 4 cases of localized bronchiectasis, 2 cases of chronic lung abscess, and 1 case of pulmonary ascariasis. Six patients (7\%) had segmentectomies due to benign lesions: centrally-located hamartochondroma in 3 patients, IgG 4-related inflammatory pseudotumor in 2 patients and bilateral arteriovenous malformations in 1 patient. The patients' characteristics are listed in Table 1.

All procedures were successfully completed by VATS with no conversion to open procedure or to lobectomy. The types of segmentectomies that were performed are illustrated in Table 2. There were 54 procedures performed in the left lung and 36 performed in the right lung. Anatomical resections in the upper lobes were the most commonly employed segmentectomies. Median operative time was 136 minutes (range, 60-280 minutes) and the mean blood loss was $50 \mathrm{~mL}$ (range, $30-250 \mathrm{~mL}$ ). Thirtytwo procedures $(36 \%)$ required less than 2 hours. The histological examination of dissected mediastinal LN (mean, $16.3 \pm 5.8$ ) showed no metastasis in all cases.

The fluorescence was detected immediately after bolus ICG injection in the central vein in all patients but had a time delay of 10-25 seconds in cases of peripheral vein administration. The median duration of intensive ICG staining was 90 seconds, regardless of the injection method. The maximum contrasting fluorescence was observed in 20-30 seconds with gradual decrease during 2 minutes. That time was enough to mark intersegmental planes. The 

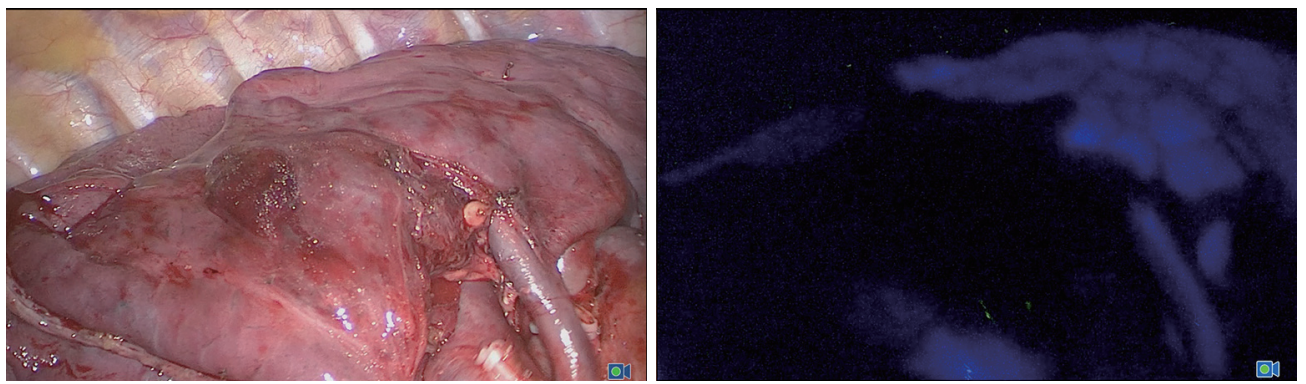

Figure 3 ICG fluorescence during right S3 segmentectomy in visible spectrum (on the left picture) and NIR spectrum (on the right picture). S3 appeared dark. S, segment; NIR, near-infrared; ICG, indocyanine green.

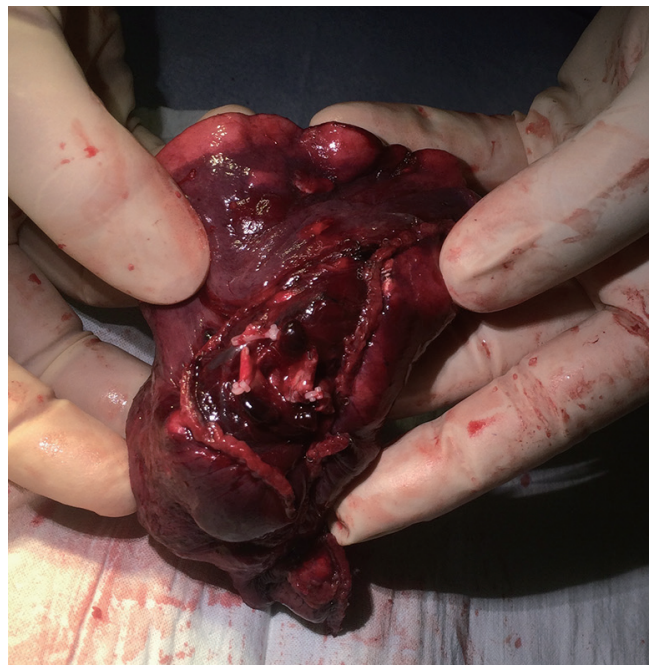

Figure 4 Right S2 segmentectomy-lung specimen (peripheral stumps of pulmonary arteries, vein, and bronchus are surrounded by stapling lines). S, segment.

further spreading of low-grade fluorescence into the target segment made it difficult to determine the boundary line.

Well-defined fluorescent borders were observed in 86 of $90(95.6 \%)$ segmentectomies. During the learning curve period, four patients had unconvincing results due to technical reasons in three cases and severe emphysema in one. The technical problems included: incomplete cessation of segmental circulation by bulldog artery clamp, mis-dividing the recurrent artery of the second segment, and the presence of multiple collateral vessels for segment three from the left. Weak fluorescence in the patient with severe emphysema and lung anthracosis required more time for marking the intersegmental planes. The additional ICG injection after identifying and dealing with the cause of failure allowed achievement of precise fluorescence. Advanced COPD in other patients did not impair the boundary line formation, but reduced the duration of intensive ICG staining. No ICGrelated complications were observed in the perioperative and postoperative period. The mean chest drain duration was $5.2 \pm 3.3$ days. Chest tubes were removed within 3 days in $32(36 \%)$ patients. Hospital length of stay ranged from 3 to 13 days. Minor complications occurred in 22 patients (24\%) and were mainly prolonged air leaks -19 patients. These patients were discharged with chest tubes attached to a Heimlich valve within 8 days. Two cases of atrial fibrillation and 1 case of pneumonia were identified in the remaining three patients with complications. No perioperative deaths were reported.

\section{Discussion}

Increased identification of small peripheral lesions as a result of implementation of low-dose CT screening programs in recent years would require novel approaches to surgical treatment. Published data suggest that thoracoscopic segmentectomy is not only a reasonable option in lung cancer patients with severe comorbidities but also the curative surgery for $\mathrm{T} 1 \mathrm{a}$ and carefully selected T1b tumors $(1,2,16)$. According to a randomized trial of Nakamura et al., segmentectomy was performed in an even broader group of lung cancer patients (17). The use of segmentectomy for pulmonary oligometastases appears to be increasing over the time (18). In addition, segmentectomies for localized infectious diseases could achieve complete lesion removal while preserving as much functional lung tissue as possible. However, this procedure is considered technically challenging, especially for VATS (3), which prevents widespread use of this method.

One of the most difficult steps of segmentectomy, besides interlobar $\mathrm{LN}$ dissection, is the identification of 

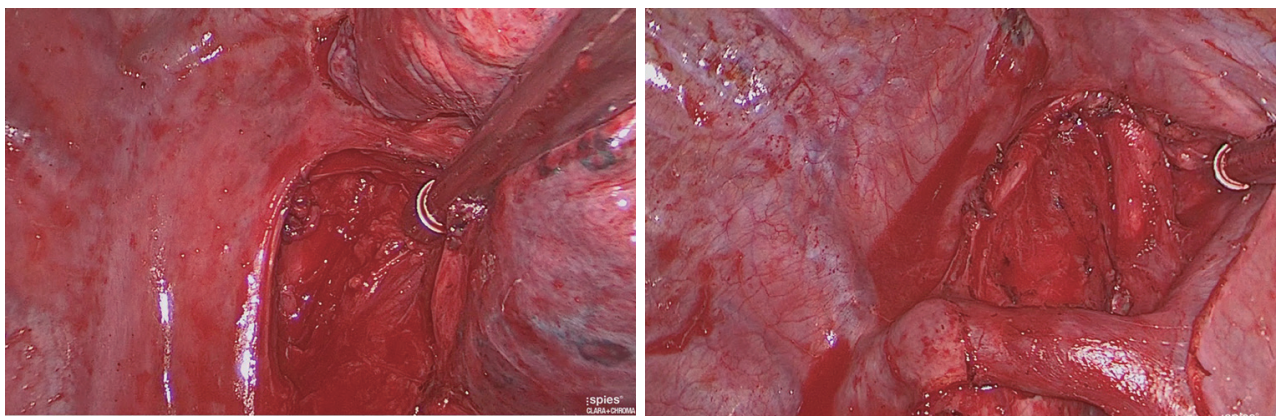

Figure 5 Lobe-specific lymphadenectomy of station 7 (on the left picture) and stations 2-4 (on the right picture).

Table 1 Characteristics in patients who underwent VATS segmentectomy $(\mathrm{n}=86)$

\begin{tabular}{|c|c|}
\hline Variable & Patients who received segmentectomy \\
\hline \multicolumn{2}{|l|}{ Sex, n [\%] } \\
\hline Male & $34[40]$ \\
\hline Female & $52[60]$ \\
\hline $\mathrm{FEV}_{1}(\%)$, mean [range] & $78.2 \pm 22.6[32-95]$ \\
\hline Smoking history (yes), n [\%] & $45[52]$ \\
\hline Size of nodule $(\mathrm{mm})$, median [range] & $19[7-51]$ \\
\hline \multicolumn{2}{|l|}{ Final pathological analysis, n [\%] } \\
\hline Infectious lesion & 16 [19] \\
\hline Benign lesion & $6[7]$ \\
\hline Median operative time (min), mean [range] & $136[60-280]$ \\
\hline Median blood loss (mL), mean [range] & $50[30-250]$ \\
\hline Median dissected mediastinal LN, mean [range] & $15[10-26]$ \\
\hline Median chest drains duration (days), mean [range] & 5 [2-21] \\
\hline Median hospital length of stay (days), mean [range] & $6[3-13]$ \\
\hline Minor complication, n [\%] & $22[24]$ \\
\hline
\end{tabular}

VATS, video-assisted thoracoscopic surgery; FEV ${ }_{1}$, forced expiratory volume in 1 second; LN, lymph node.

intersegmental planes, which is essential both for oncological and lung-sparing reasons. The wrong interpretation of the invisible border between adjacent segments could potentially lead to unnecessary lung resection, positive surgical margins and even loss the target in the remaining lung parenchyma. In this regard, technical assistance for intersegmental plane formation during thoracoscopic segmentectomy is crucial.

In recent decades, various methods that can be used to identify intersegmental planes have been published $(4,13,19-21)$. The commonly used test involves inflation the lung after clamping the target bronchus in order to create a boundary line between the inflated lung and deflated target 
Table 2 Types of segmentectomy $(\mathrm{n}=90)$

\begin{tabular}{|c|c|}
\hline Types & Number [\%] \\
\hline Right lung & $36[40]$ \\
\hline Upper lobe & 21 [23] \\
\hline $\mathrm{S} 2$ & 8 [9] \\
\hline $\mathrm{S} 1+2$ & $6[7]$ \\
\hline S1 & $5[5]$ \\
\hline S3 & 2 \\
\hline Lower lobe & $15[17]$ \\
\hline S6 & 9 [10] \\
\hline$S 7-10$ & $4[5]$ \\
\hline S8 & 1 \\
\hline S9+10 & 1 \\
\hline Left lung & 54 [60] \\
\hline Upper lobe & $40[44]$ \\
\hline $\mathrm{S} 1+2$ & $14[16]$ \\
\hline $\mathrm{S} 4+5$ & $12[13]$ \\
\hline S1-3 & $11[12]$ \\
\hline S3 & 2 \\
\hline $\mathrm{S} 2$ & 1 \\
\hline Lower lobe & $11[12]$ \\
\hline S6 & 7 [8] \\
\hline S8-10 & $3[3]$ \\
\hline S10 & 1 \\
\hline Poly-segmentectomy & $3[3]$ \\
\hline$S 1+2+S 6$ & 1 \\
\hline$S 4+5+S 8-10$ & 1 \\
\hline$S 4+5+S 8$ & 1 \\
\hline
\end{tabular}

S, segment.

segment. However, excessive lung inflation may spread the air through interalveolar pores, shift the intersegmental border and also obstruct the view during the VATS. Other methods keep the target segment inflated by ligating the bronchus before whole lung deflation (4), or by selective bronchial jet ventilation (19), or by air injection into the bronchus through a butterfly needle. These are effective in securing a clear operative field, but all share the same limitations as previously described methods.

Other experts developed a methylene blue staining technique through the bronchial path following the feasibility of the method in vivo (20). The same administration method was described by $\mathrm{Oh}$ et al. (13) using another dye-ICG. In these clinical trials, the accuracy of revealing borders is clearly shown, but intrabronchial dyes have other limitations. First of all, the additional need for hilar structure manipulation strongly affects the dye spreading. Furthermore, careful selection of the dose of dye is necessary, since using an excessive amount of dye leads to unclear borders and difficult resection. In addition, this method requires meticulous sputum suction before the bronchial injection to prevent incomplete target segment staining caused by sputum blockage.

Sugimoto et al. in animal studies (21) demonstrated that intersegmental lines can be clearly detected by direct injection of indigo carmine into the target segmental artery. The idea is good but attention must be drawn to a number of limitations. This method seems to be very challenging in real clinical practice due to excessive manipulation of small pulmonary arteries. Additionally, some segments had blood supply from several artery branches, so one needs to inject the dye into all these stumps.

The use of perfusion-based methods for delineating lung segment borders has become an innovative solution. ICGfluorescence has become possible in the last few years (10-14). This method doesn't require intraoperative lung ventilation which significantly improves visualization during VATS especially in cases of advanced emphysema. After confirming reproducibility of ICG fluorescence in experimental animal models (22), a group of Japanese scientists described successful visualization of an intersegmental plane in 8 patients (12). With increased experience the frequency of inconclusive results decreased from $15.4 \%$ (14) to $1.6 \%$ (10). In our study clear identification of the intersegmental plane was reported in 86 out of 90 cases (95.6\%). However, only one was associated with poor fluorescence quality, while the remaining three cases were due to technical mistakes. As described in prior studies, advanced emphysema was the cause of poor fluorescence. Other investigators (13) showed that longer smoking history, lower $\mathrm{FEV}_{1}$, and lower parenchymal density on $\mathrm{CT}$ scan impair the quality of glow. On the other hand, perfusionbased methods were widely used in cases of severe bullous emphysema previously (23) and still investigated now (9). So, it seems to us quite strange to blame emphysema, if one has some perfusion issues due to this. In our experience, advanced emphysema that decreases ICG-fluorescence intensity requires a higher dose of ICG, rather than use of a different method.

The intensity and duration of fluorescence depend on the method of injection $(13,24)$, and on the optical device 
used for detection of fluorescence (11). Sugimoto et al. (21) suggest that using both peripheral vein and segmental bronchus ICG injection allows identification of the intersegmental plane. However, transbronchial staining requires a higher dose of ICG.

In current work, we used intravenous ICG administration given technical simplicity and a lower required dose of the agent. In prior studies, it was shown that the frequency of allergic reactions with ICG doses below $0.5 \mathrm{mg} / \mathrm{kg}$ was reported at $0.003 \%$, but significantly increases if the dose exceeds $5 \mathrm{mg} / \mathrm{kg}$ (25). We used a dose of $0.15 \mathrm{mg} / \mathrm{kg}$ in all cases but 11 (in which the total dose was $0.3 \mathrm{mg} / \mathrm{kg}$ ). No adverse reactions were observed. It is worth noting that some investigators use higher doses of ICG with intravenous methods. For instance, Misaki et al. administered ICG at a dose of $3 \mathrm{mg} / \mathrm{kg}$ as described in their earlier work (12). In another study, the same group of authors describes inadequate results with the lower dose of $0.5 \mathrm{mg} / \mathrm{kg}$ (14). Kasai et al. (11) compared optical devices, which differed in the spectrum of light recognition and the method of image production. When using two wave spectrum analysis, the optical device is able to recognize the difference between a 940 -nm wave and an 805 $\mathrm{nm}$ wave. A single wave device emits a $780-\mathrm{nm}$ wave, while detecting an 830-nm wave, producing a fluorescent image in perfused tissue. Kasai et al. speculated that using single wave optical devices allows use of a reduced dose of ICG to $0.5 \mathrm{mg} / \mathrm{kg}$ and increased fluorescence length from 220 to 370 seconds. Because of that, we used a single-wave optical device for producing the image. The length of maximal fluorescence in our study did not go above 130 seconds. Using the dose of ICG lower than described in previous works did not interfere with clear intersegmental plane detection.

Looking at our experience we would suggest ICG-perfusion method as the best option for VATS segmentectomy in terms of identification of intersegmental planes, evaluation of pulmonary vasculature, avoiding the need of ventilation tests. The fluorescence creates an augmented reality that might also be used for LN mapping and detection of pulmonary lesions.

\section{Conclusions}

The ICG-fluorescence technology is safe and effective for verification of anatomic segment borders for VATS. The perfusion-based ICG fluorescence technique has advantages for thoracoscopic surgeries compared to other methods. Doubling the dose of ICG allows clear detection of the intersegmental plane, even in certain suboptimal conditions.

\section{Acknowledgements}

The authors thank Aleksandr Obornev and Eugeniy Zinchenko for their assistance in the research.

\section{Footnote}

Conflicts of Interest: The authors have no conflicts of interest to declare.

Ethical Statement: The study was approved by the Institutional Review Board (No. 2015-27) and written informed consent was obtained from all patients.

\section{References}

1. Aokage K, Yoshida J, Hishida T, et al. Limited resection for early-stage non-small cell lung cancer as functionpreserving radical surgery: a review. Jpn J Clin Oncol 2017;47:7-11.

2. Hwang Y, Kang CH, Kim HS, et al. Comparison of thoracoscopic segmentectomy and thoracoscopic lobectomy on the patients with non-small cell lung cancer: a propensity score matching study. Eur J Cardiothorac Surg 2015;48:273-8.

3. Yang CF, D'Amico TA. Thoracoscopic segmentectomy for lung cancer. Ann Thorac Surg 2012;94:668-81.

4. Oizumi H, Kato H, Endoh M, et al. Techniques to define segmental anatomy during segmentectomy. Ann Cardiothorac Surg 2014;3:170-5.

5. Rino Y, Yukawa N, Sato T, et al. Visualization of blood supply route to the reconstructed stomach by indocyanine green fluorescence imaging during esophagectomy. BMC Med Imaging 2014;14:18.

6. Yamashita S, Tokuishi K, Miyawaki M, et al. Sentinel node navigation surgery by thoracoscopic fluorescence imaging system and molecular examination in non-small cell lung cancer. Ann Surg Oncol 2012;19:728-33.

7. Nomori H, Cong Y, Sugimura H. Utility and pitfalls of sentinel node identification using indocyanine green during segmentectomy for cT1N0M0 non-small cell lung cancer. Surg Today 2016;46:908-13.

8. Kim HK, Quan YH, Choi BH, et al. Intraoperative pulmonary neoplasm identification using near-infrared fluorescence imaging. Eur J Cardiothorac Surg 2016;49:1497-502.

9. Li H, Zhou J, Chi C, et al. Clinical application of near-infrared thoracoscope with indocyanine green in video-assisted thoracoscopic bullectomy. J Thorac Dis 
2016;8:1841-5.

10. Iizuka S, Kuroda H, Yoshimura K, et al. Predictors of indocyanine green visualization during fluorescence imaging for segmental plane formation in thoracoscopic anatomical segmentectomy. J Thorac Dis 2016;8:985-91.

11. Kasai Y, Tarumi S, Chang SS, et al. Clinical trial of new methods for identifying lung intersegmental borders using infrared thoracoscopy with indocyanine green: comparative analysis of 2- and 1-wavelength methods. Eur J Cardiothorac Surg 2013;44:1103-7.

12. Misaki N, Chang SS, Igai H, et al. New clinically applicable method for visualizing adjacent lung segments using an infrared thoracoscopy system. J Thorac Cardiovasc Surg 2010;140:752-6.

13. Oh S, Suzuki K, Miyasaka Y, et al. New technique for lung segmentectomy using indocyanine green injection. Ann Thorac Surg 2013;95:2188-90.

14. Tarumi S, Misaki N, Kasai Y, et al. Clinical trial of videoassisted thoracoscopic segmentectomy using infrared thoracoscopy with indocyanine green. Eur J Cardiothorac Surg 2014;46:112-5.

15. Pischik VG. Technical difficulties and extending the indications for VATS lobectomy. J Thorac Dis 2014;6:S623-30.

16. Sihoe AD, Van Schil P. Non-small cell lung cancer: when to offer sublobar resection. Lung Cancer 2014;86:115-20.

17. Nakamura K, Saji H, Nakajima R, et al. A phase III randomized trial of lobectomy versus limited resection for small-sized peripheral non-small cell lung cancer (JCOG0802/WJOG4607L). Jpn J Clin Oncol

Cite this article as: Pischik VG, Kovalenko A. The role of indocyanine green fluorescence for intersegmental plane identification during video-assisted thoracoscopic surgery segmentectomies. J Thorac Dis 2018;10(Suppl 31):S3704S3711. doi: 10.21037/jtd.2018.04.84
2010;40:271-4.

18. Berry MF. Role of segmentectomy for pulmonary metastases. Ann Cardiothorac Surg 2014;3:176-82.

19. Okada M, Mimura T, Ikegaki J, et al. A novel video-assisted anatomic segmentectomy technique: selective segmental inflation via bronchofiberoptic jet followed by cautery cutting. J Thorac Cardiovasc Surg 2007;133:753-8.

20. Zhang Z, Liao Y, Ai B, et al. Methylene blue staining: a new technique for identifying intersegmental planes in anatomic segmentectomy. Ann Thorac Surg. 2015;99:238-42.

21. Sugimoto S, Oto T, Miyoshi K, Miyoshi S. A novel technique for identification of the lung intersegmental plane using dye injection into the segmental pulmonary artery. J Thorac Cardiovasc Surg 2011;141:1325-7.

22. Misaki N, Chang SS, Gotoh M, et al. A novel method for determining adjacent lung segments with infrared thoracoscopy. J Thorac Cardiovasc Surg 2009;138:613-8.

23. Gotoh M, Okamoto T, Yamamoto Y, et al. Real time imaging and quantitative evaluation of the emphysematous lung by infrared thoracoscopy in experimental dogs. ASAIO J 2005;51:148-51.

24. Sekine Y, Ko E, Oishi H, et al. A simple and effective technique for identification of intersegmental planes by infrared thoracoscopy after transbronchial injection of indocyanine green. J Thorac Cardiovasc Surg 2012;143:1330-5.

25. Speich R, Saesseli B, Hoffmann U, et al. Anaphylactoid reactions after indocyanine-green administration. Ann Intern Med 1988;109:345-6. 